\title{
DOMINIO DEL CONCEPTO DE RESPONSABILIDAD SOBRE LA PRESENTACIÓN FINANCIERA POR LOS ESTUDIANTES DE DOS UNIVERSIDADES DE LA CIUDAD DE CALI*
}

\author{
DOMAIN OF THE CONCEPT OF RESPONSIBILITY FOR THE \\ FINANCIAL PRESENTATION BY THE STUDENTS OF TWO \\ UNIVERSITIES OF THE CITY OF CALI, COLOMBIA \\ DOMÍNIO DO CONCEITO DE RESPONSABILIDADE NA \\ APRESENTAÇÃO FINANCEIRA DOS ALUNOS DE DUAS \\ UNIVERSIDADES DA CIDADE DE CALI \\ DOMAINE DU CONCEPT DE RESPONSABILITÉ SUR LA \\ PRÉSENTATION FINANCIĖRE PAR LES ÉTUDIANTS DE DEUX \\ UNIVERSITÉS DE LA VILLE DE CALI
}

\author{
BIBIANA RENDÓN ÁLVAREZ† \\ EDILBERTO MONTAÑO OROZCO \\ XIMENA SÁNCHEZ MAYORGA§
}

Fecha de recepción: 10 de julio de 2020

Fecha de aprobación: 19 de septiembre de 2020

* Este artículo es resultado del proyecto de investigación "Conocimiento respecto de la responsabilidad en la preparación y presentación de los estados financieros en los estudiantes de pregrado de los programas académicos de contaduría pública y administración. Caso de estudio en dos universidades de la ciudad de Santiago de Cali".

† Contadora pública, Universidad del Valle, Cali, Colombia; magíster en Administración, Universidad del Valle, Cali; profesora asociada, Universidad del Valle, Cali; integrante del grupo de investigación en Previsión y Pensamiento Estratégico, Universidad del Valle; integrante del grupo de investigación Gestión y Productividad Contable, Universidad Libre. Sede San Fernando, Cali. bibiana.rendon@correounivalle.edu.cohttps://orcid.org/0000-0002-2635-0557.

‡ Contador público, Universidad de San Buenaventura, Cali, Colombia; magíster en Derecho constitucional, Universidad Libre, Cali; especialista en Finanzas, Universidad del Valle, Cali; profesor titular, Universidad del Valle, Cali, Sede San Fernando. edillberto. montano@correounivalle.edu.co - https://orcid.org/0000-0002-7168-6797.

$\S$ Contadora pública, Universidad del Valle, Cali, Colombia; magíster en Administración de Empresas, Universidad del Valle, Cali; especialista en informática educativa, Universidad Libre, Cali; doctorante en Educación Mediada, Universidad de la Salle, San José, Costa Rica; directora, Programa de Contaduría Pública y profesora, Universidad Libre; líder del grupo de investigación Gestión y Productividad Contable, Universidad Libre, Cali. ximena.sanchez@unilibre.edu.co - https://orcid.org/00000001-951 1-4905.

Criterio Libre N. ${ }^{\circ} 33$ Bogotá (Colombia) Julio-Diciembre 2020 pp. $179-200$ ISSN 1900-0642 ISSN electrónico 2323-0886 


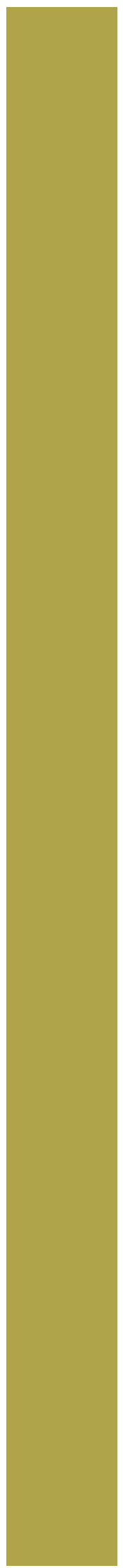

RESUMEN

El presente artículo pretende clarificar el concepto de la responsabilidad en la preparación, elaboración y presentación de estados financieros en las empresas y su relación con quienes tienen a su cargo la representación legal (administrador) y el contador público, así como valorar el grado de apropiación del tema por los estudiantes de los programas académicos de pregrado de Administración de Empresas y Contaduría Pública de dos universidades de la ciudad de Santiago de Cali.

Se incluyen aspectos teóricos, normativos y conceptuales que permiten, tanto a la comunidad académica vinculada a las ciencias administrativas y económicas, como a profesionales afines a los negocios, comprender, cumplir con la normativa respectiva y precisar la responsabilidad inherente sobre esta exigencia legal. Para la valoración del dominio del tema, se aplicaron encuestas a estudiantes de dichos programas académicos. El estudio concluye que los estudiantes de los programas académicos de pregrado de Administración de Empresas y Contaduría Pública de las mencionadas instituciones desconocen o no tienen claridad respecto al papel fundamental que les compete en la preparación y presentación de estados financieros y el marco normativo aplicable.

PALABRAS CLAVE:

administrador, certificación de estados financieros, estados financieros, responsabilidad.

CLASIFICACIÓN JEL: M100, M400, M410, M480.

This article aims to clarify the concept of responsibility in the preparation, elaboration and presentation of financial statements in companies and their relationship with those in charge of legal representation (administrator) and the public accountant, as well as assess the degree of appropriation of the subject by students of the undergraduate academic programs of Business Administration and Public Accounting of two universities in the city of Santiago de Cali, Colombia.

It includes theoretical, normative and conceptual aspects that allow, both the academic community linked to the administrative and economic sciences, and professionals related to business, to understand, comply with the respective regulations and specify the inherent responsibility 
for this legal requirement. For the assessment of the domain of the subject, surveys were applied to students of said academic programs. The study concludes that the students of the undergraduate programs of Business Administration and Public Accounting of the aforementioned institutions are unaware of or lack clarity regarding their fundamental role in the preparation and presentation of financial statements and the applicable regulatory framework.

Keywords: administrator, certification of financial statements, financial statements, accountability.

JEL classification: M100, M400, M410, M480.

\section{RESUMO}

Este artigo procura clarificar o conceito de responsabilidade na preparação, elaboração e apresentação de demonstrações financeiras nas empresas e a sua relação com os responsáveis pela representação legal (administrador) e o contabilista público, e também avaliar o grau de apropriação deste tema pelos estudantes dos programas académicos de graduação em Administração de Empresas e Contabilidade Pública de duas universidades da cidade de Santiago de Cali, Colômbia.

Inclui aspectos teóricos, normativos e conceptuais que permitem tanto à comunidade académica ligada às ciências administrativas e económicas, como aos profissionais relacionados com as empresas, compreender, cumprir os respectivos regulamentos e especificar a responsabilidade inerente a este requisito legal. A fim de avaliar o domínio da matéria, foram aplicados inquéritos aos estudantes destes programas académicos. $\bigcirc$ estudo conclui que os estudantes dos programas académicos de licenciatura em Administração de Empresas e Contabilidade Pública das referidas universidades não conhecem ou não são claros quanto ao seu papel fundamental na preparação e apresentação das demonstrações financeiras e do quadro regulamentar aplicável.

Palavras-chave: administrador, certificação de demonstrações financeiras, demonstrações financeiras, responsabilidade.

Classificação JEL: M100, M400, M410, M480.

\section{RÉSUMÉ}

Cet article tente de clarifier le concept de responsabilité dans la préparation, l'élaboration et la présentation des états financiers dans les entreprises et leur relation avec les personnes chargées de la représentation légale (administrateur) et l'expert-comptable, et également d'évaluer le degré d'appropriation de ce sujet par les étudiants des programmes 
académiques de premier cycle en administration des affaires et en comptabilité publique de deux universités de la ville de Santiago de Cali, en Colombie.

Il comprend des aspects théoriques, normatifs et conceptuels qui permettent à la fois à la communauté académique liée aux sciences administratives et économiques, ainsi qu'aux professionnels liés aux affaires, de comprendre, de se conformer aux réglementations respectives et de préciser la responsabilité inhérente à cette exigence légale. Afin d'évaluer le domain du sujet, des enquêtes ont été appliquées aux étudiants de ces programmes académiques. L'étude conclut que les étudiants des programmes académiques de premier cycle en administration des affaires et en comptabilité publique des universités mentionnées ne connaissent pas ou ne sont pas clairs quant à leur rôle fondamental dans la préparation et la présentation des états financiers et du cadre réglementaire applicable.

Mots clés: administrateur, certification des états financiers, états financiers, responsabilité.

Classification JEL: M100, M400, M410, M480.

\section{INTRODUCCIÓN}

Este artículo aborda la problemática sobre la transparencia en la información financiera, en la que se incluye el cumplimiento legal de presentación de los estados financieros de propósito general, como fuente para tomar decisiones, con el antecedente de distintas investigaciones en donde se menciona la importancia de esta observancia (Alfredo \& Becerra, 2010; Carolina \& Sánchez, 2013 ; Garza Sánchez, Cortez Alejandro, Méndez Sáenz, \& Rodríguez García, 2017; Rafael, 2007). Igualmente, en los distintos conceptos que se han generado por parte del Consejo Técnico de la Contaduría (2021) desde el año 2013 hasta la fecha se han emitido 707 conceptos relacionados con responsabilidad, firma y presentación de estados financieros, en los que dan respuesta a estos temas. No obstante, no se encuentran investigaciones a nivel nacional que presenten el tema abordado conforme a la normatividad vigente, ni a nivel de los profesionales y mucho menos a nivel de la apropiación de estos conceptos en la formación de los estudiantes de administración de empresas y de contaduría pública.

Dado lo anterior, este artículo tiene como propósito indagar a estudiantes de pregrado de administración de empresas y contaduría pública acerca del conocimiento que tienen, sobre quién recae la responsabilidad en la preparación y presentación de estados financieros de las sociedades 
mercantiles y la importancia de comprender el rol que desempeñan el administrador 0 representante legal, el profesional contable en calidad de contador público o de revisor fiscal, en la emisión de información financiera y poder así generar estrategias que desde la formación se impartan y apropien de lo anterior.

Dada la importancia de la información financiera en la toma de decisiones por diversos usuarios - stakeholders- y el alcance y responsabilidades que pueden sobrevenir por inadecuada presentación, clasificación o revelación, los planes curriculares de los programas académicos de administración de empresas y contaduría pública de las universidades Libre y del Valle, contemplan en las asignaturas de contabilidad, legislación empresarial o Derecho comercial, cursadas en diversos semestres, aspectos relacionados con la preparación de información contable y su respectiva presentación y revelación por medio de los estados financieros. Sin embargo, el proceso o didáctica mediante el cual los estudiantes aprenden y/o se apropian de dichos conceptos y se forman, es variado.

Sobre lo anterior es pertinente referenciar lo que Epstein \& Birchard (2001) plantean:

Muy pocos directores comprenden plenamente el valor de rendir cuentas claras. No pueden definir este concepto con claridad ni aplicarlo para conseguir ventajas en el día a día ni a largo plazo. Sin embargo, la responsabilidad y la trasparencia pueden dar a una organización una ventaja competitiva en la ejecución de su estrategia corporativa y a la hora de ayudar a los individuos, equipos y divisiones a conseguir un rendimiento inesperado. (Epstein y Birchard, 2001, p. 18).

El Código de Comercio (Presidencia de la República, 1971) y la Ley 222 de 1995 (Congreso de Colombia, 1995) establecen los deberes y obligaciones de los comerciantes (persona jurídicas o naturales), régimen de administradores, el cumplimiento respecto a los libros de comercio y de preparación e información financiera, de obligatoria observancia, que se traduce en tener un adecuado conocimiento de ello por parte de los responsables.

Según el artículo 22 de la Ley 222 de 1995 (Congreso de Colombia, 1995), son administradores' el representante legal, el liquidador, los miembros de juntas o consejos directivos y quienes, de acuerdo con los estatutos, ejerzan dicha función ${ }^{2}$. Para los contadores públicos y representante legal, la citada norma estipula la certificación de estados financieros.

\section{- MARCO DE REFERENCIA}

\subsection{MARCO TEÓRICO Y CONCEPTUAL}

\subsubsection{Teorías administrativas}

Hoy día siguen vigentes algunos aspectos importantes de las teorías clásicas de la organización, entre las que se destaca la administración industrial y general, descrita por Henry Fayol en su obra La administración industrial, publicada en 1916 (Murillo Vargas et al., 20111 . Esta considera que la empresa puede estar dividida en seis operaciones: técnicas, comerciales, financieras, seguridad, contables y administrativas.

Se resalta esta teoría para la investigación presentada, dada la importancia de la operación contable en la que se involucran algunos elementos como inventario, el balance, el precio

1 El concepto de administrador que se aborda en este artículo es desde lo legal, el cual apunta a lo orgánico en las sociedades.

2 En cuanto a los gerentes, no siempre tienen la calidad de representantes legales; sin embargo, cada sociedad determinará la responsabilidad y funciones de cada una de las personas que se designen como tales. 
... la contabilidad permite a

las organizaciones conocer el

resultado financiero para tomar

decisiones y prever situaciones

futuras, lo cual se obtiene mediante

los estados financieros; por tanto,

debe atenderse adecuadamente lo

operativo y lo legal, dado

que en Colombia existe un marco

regulatorio relacionado

con las obligaciones de

las sociedades mercantiles. de costo y la estadística; además teniendo en cuenta que se consideran las operaciones de contabilidad como "[...] un medio por el cual ayuda a completar el análisis de la situación de la empresa, en suma plantea que la contabilidad debe poseer dos características: claridad en la información y sencillez para su manejo" (Murillo Vargas et al., 2011 , p. 204).

Dado lo anterior, la contabilidad permite a las organizaciones conocer el resultado financiero ${ }^{3}$ para tomar decisiones y prever situaciones futuras, lo cual se obtiene mediante los estados financieros; por tanto, debe atenderse adecuadamente lo operativo y lo legal, dado que en Colombia existe un marco regulatorio relacionado con las obligaciones de las sociedades mercantiles.

Siendo la contabilidad una operación dentro de la organización, deben ser los administradores los encargados de velar por su realización y cumplimiento, lo cual estaría vinculado dentro del tipo de ética de la responsabilidad planteado por Max Weber (Murillo Vargas et al., 2011 ), entendido como:

La acción, por el contrario, está motivada y guiada por una escogencia racional y atinada, un discernimiento entre lo que es realista $\circ$ no, de conformidad o no con los medios accesibles y que conlleva unas consecuencias que se pueden o no asumir. (Aktouf, 2001, p. 93)

A pesar de la mencionada función de los administradores, la elaboración de la

3 Este artículo aborda solamente el estándar financiero; no obstante, las organizaciones deberían considerar otros modelos contables como la biocontabilidad y la sociocontabilidad, lo cual se plantea en la obra Teoría tridimensional de la contabilidad (Mejía Soto, Montilla Galvis, Montes Salazar y Mora Roa, 2014). 
contabilidad estará a cargo de un contador público, que es el profesional idóneo designado para tal fin.

\subsubsection{Teoría de la agencia}

Jensen y Meckilng (1976, p. 308) definen "relación de agencia como un contrato, en el cual una o más personas (el principal) contratan a otras personas (el agente) para que realicen determinado servicio a su nombre, lo que implica un grado de delegación de autoridad del principal al agente". Conforme a esta teoría se plantea la existencia de diversos agentes, con un objetivo de maximización del beneficio, y además que la información debe ser pública y completa; siendo así los estados financieros un requisito que permite presentar los resultados obtenidos por una organización.

Según Ryan, Scapens y Theobald (2004), la teoría de la agencia ha permitido el desarrollo de la teoría de la contabilidad positiva, pues se reconoce la relación costo-beneficio de la información para motivar la toma de decisiones de los agentes.

Además, es necesario que se establezcan mecanismos de incentivos y control formalizados en los contratos y en los que se implementan sistemas de información que permiten al principal conocer lo que ha realizado el agente; con relación al presente artículo la importancia de los estados financieros de propósito general, los cuales contribuyen a la toma de decisiones.

\subsubsection{Teoría de la contabilidad y el control}

Para Sunder (1944) las organizaciones son un conjunto de contratos en las que el suministro de información permite el diseño y la ejecución de estos, en tanto que el control en estas permite el equilibrio entre todos los participantes. Igualmente, considera las funciones de la contabilidad para posibilitar el funcionamiento de la empresa, encontrando dos de ellas que se relacionan con la problemática abordada en el presente artículo, así: "3. Informar a los agentes apropiados hasta qué punto los otros agentes han cumplido sus obligaciones contractuales y han recibido sus derechos [...]; 5. Provee una base de conocimiento común de información verificada a todos los participantes para facilitar la negociación y la formalización de los contratos" (1944, p. 47). Esto representa que el sistema de contabilidad genera estados financieros de propósito general como

... base común de información en forma de revelación pública. La publicación de los estados financieros, la revelación de las políticas contables y los detalles significativos en las notas, los análisis administrativos de los estados financieros y los resultados, e incluso las previsiones financieras, tienen el efecto de reducir las sorpresas en el momento de la renegociación del contrato. (1944, pp. 56 y 571

Lo anterior se vincula al paradigma de la utilidad, puesto que aborda como concepto principal, que el propósito y objetivos de los estados financiera es el de suministrar información útil a los usuarios (Pereda, 1990). Es preciso, que la teoría contable aborde un marco de referencia que permita satisfacer las necesidades de los diferentes usuarios, lo que afirma Pereda (1 990) al considerar que "bajo este paradigma el contenido de los estados financieros se orienta a cubrir las necesidades de los usuarios; constituye por lo tanto la información un soporte vital para la 'adecuada' toma de decisiones". (Citado por Montes Salazar, Mejía Soto, \& Valencia Salazar, 2006, p. 83).

\subsubsection{Código de buen gobierno y accountability}

El gobierno corporativo es un conjunto de principios, valores y buenas prácticas empresariales, que permiten que las organizaciones sean eficientes y transparentes, lo que da lugar a promover 
acciones en materia contable que permitan a los administradores controlar su gestión y verificar el cumplimiento de las políticas contables.

Según la Organización para la Cooperación y el Desarrollo Económicos (OCDE, 2004), un punto por considerar es la divulgación de datos y transparencia, en la que se incluyen los informes financieros oportunos como fuente de información comúnmente utilizada. Epstein \& Birchard (2001) plantean que: "Muy pocos directores comprenden plenamente el valor de rendir cuentas claras. No pueden definir este concepto con claridad ni aplicarlo para conseguir ventajas en el día a día ni a largo plazo". Citando a Handy, C. (1994), precisan que "contar hace las cosas visibles, y el hecho de contarlas hace que las cosas cuenten" (Epstein \& Birchard, 2001, p. 18).

La rendición de cuentas tiene que ver con instituciones modernas eficientes, con personal técnico bien entrenado. Pero también tiene que ver con mecanismos financieros y contable modernos y apropiados. De ahí que el Banco Mundial, por ejemplo, está trabajando en diversos continentes en mejorar los sistemas de presupuesto, de contabilidad y de información; está contribuyendo, también, a formar cuadros de contabilistas y auditores de altas calidades profesionales, y está ayudando a fortalecer el marco legal que permitirá la existencia de prácticas modernas de contabilidad. Y para ello está realizando evaluaciones periódicas y sistemáticas sobre lo que denomina Financial Accountability. (Cepeda, 2004, p. 87)

En Colombia, no todas las empresas están obligadas a adoptar un código de buen gobierno, pero la Superintendencia de Sociedades, Confecamaras y la Cámara de Comercio de Bogotá (2009) expidieron la Guía de gobierno corporativo para sociedades cerradas y de familia. En esta guía se describen los mínimos legales y contables que con mayor frecuencia incumplen las sociedades cerradas, que son problemas recurrentes que deben ser solucionados y conocidos por los empresarios; estos son en materia contable: la administración no verifica el cumplimiento de los requisitos legales en materia contable; las irregularidades, en materia contable, más representativas detectadas fueron la contabilidad atrasada, falta de soportes contables, entre otros.

\subsubsection{Contabilidad}

Los administradores de las sociedades mercantiles deben concebir la contabilidad "como un sistema de información que interactúa en las distintas áreas funcionales y operativas de las empresas, reconoce, mide y revela hechos económicos a partir de criterios previamente definidos" (Montaño y Rendón, 2017, p. 43). Lo anterior se evidencia en las obligaciones de las sociedades comerciales, que, según el artículo 19 del Código de Comercio (Presidencia de la República, 1971), estipula el deber de llevar contabilidad.

En cuanto a las reglamentaciones vigentes para llevar la contabilidad, la Ley 1314 de 2009 (Congreso de Colombia, 2009) determina que en Colombia todo aquel que está obligado a llevarla debe acogerse al marco técnico normativo contable del Decreto 2420 de 2015 , lo que significa que las sociedades mercantiles deben determinar el grupo al cual pertenecen, para así aplicar el reglamento correspondiente.

Son tres los grupos de aplicación; la mayoría de las sociedades comerciales están delimitadas dentro de los grupos 2 y 3, por lo que deberán aplicar las normas correspondientes de conformidad con la vigencia de las mismas por cada período contable.

\subsubsection{Estados financieros}

En cuanto a la obligatoriedad en preparar y difundir estados financieros, el artículo 34 de 
la Ley 222 de 1995 (Congreso de Colombia, 1995) estipula que, al final de cada período anual, las sociedades tienen dicha exigencia, y que las mismas deben estar debidamente certificadas.

Con lo anterior se establece que sobre los administradores recae directamente dicho compromiso y que son estos los responsables de las obligaciones de la sociedad.

Así mismo, la responsabilidad en la preparación y organización de la información en términos contables es del contador público, quien es el encargado de reconocer, medir y revelar, conforme a las políticas definidas por los administradores de acuerdo al marco técnico normativo contable.

Igualmente el Código de Comercio expedido en 1971 (Presidencia de la República, 1971), incluyó en el capítulo VIII del Libro II, lo correspondiente al revisor fiscal; encontrándose que el artículo 203 estipula las sociedades obligadas a tener dicha figura. También, la Ley 43 de 1990 (Congreso de la República, 1990), en el parágrafo segundo del artículo 13 , menciona que será obligatorio tener revisor fiscal en todas las sociedades comerciales, de cualquier naturaleza, cuyos activos brutos al 31 de diciembre del año inmediatamente anterior sean o excedan el equivalente de cinco mil salarios mínimos y/o cuyos ingresos brutos durante el año inmediatamente anterior sean o excedan al equivalente a tres mil salarios mínimos.

Como se puede evidenciar, el revisor fiscal emite opinión respecto del cumplimiento de las obligaciones que, en materia de contabilidad y presentación de estados financieros, tienen las organizaciones.

La Ley 60 de 1981 (Congreso de Colombia, 1981 ) reconoce la disciplina de administración de empresas como profesión y dicta las normas sobre el ejercicio de la misma en Colombia; el artículo 4 estipula que para ejercerla se debe

\section{... la responsabilidad en la}

preparación y organización de la

información en términos contables

es del contador público, quien es

el encargado de reconocer, medir

y revelar, conforme a las políticas

definidas por los administradores

de acuerdo al marco técnico

normativo contable. 
No obsta agregar que la mencionada ley [145 de 1960] no establece que se requiera tal condición -profesional en Administración de Empresas-para ostentar la calidad de representante legal, excepto que los estatutos, disposición interna o convocatoria para proveer ciertos cargos lo determine. Es de esperarse que para algunos cargos de administración o dirección empresarial se cuente con formación en esta disciplina. contar con título universitario expedido por una institución de educación superior aprobada por el gobierno nacional y con matrícula profesional que expide el Consejo Profesional de Administración de Empresas.

Cabe indicar que para Molina (2006), el concepto de administrador que es abordado en la Ley 60 de 1981 (Congreso de Colombia, 19811 invoca cuestiones funcionales, al considerar que del mismo hacen parte "[...] todas y cada una de las personas que, al interior o en relación con la sociedad, cumplen funciones de administrador" (p. 63); no obstante, el concepto abordado en el presente artículo es desde el aspecto legal, contemplado en el artículo 22 de la Ley 222 de 1995 (Congreso de Colombia, 1995).

En cuanto a los tipos de estados financieros determinados en la Ley 222 de 1995 (Congreso de Colombia, 1995), están los consolidados, los certificados y los dictaminados. Los estados financieros consolidados son preparados y presentados, según el artículo 35 de la norma citada, por las empresas que son controlantes de otras; el artículo 37 determina que los estados financieros certificados deben ir acompañados de certificación expedida por el representante legal y el contador, en la cual declaren que se han verificado las afirmaciones contenidas en ellos y que las cifras son fieles a la contabilidad; el artículo 38 define los estados financieros dictaminados como aquellos que se acompañan de la opinión profesional del revisor fiscal o, a falta de este, de un contador público independiente.

Por último, los estados financieros que deben presentar las sociedades en Colombia, conforme a lo dispuesto en el artí́culo 2 de la Ley 1314 de 2009 (Congreso de Colombia, 2009), y de acuerdo con los tres grupos en que fueron clasificadas mediante decretos expedidos por el gobierno nacional durante los años 2012 a 2014, los cuales se compilaron en el Decreto Único Reglamentario -DUR- 2420 de 2015 , se presentan en la tabla 1. 
Tabla 1. Conjunto o juego completo de estados financieros.

\begin{tabular}{|c|c|c|c|}
\hline Criterio & Grupo 1 & Grupo 2 & Grupo 3 \\
\hline $\begin{array}{l}\text { Conjunto o juego } \\
\text { completo de estados } \\
\text { financieros al final del } \\
\text { período }\end{array}$ & $\begin{array}{l}\text { a. Estado de situación } \\
\text { financiera. } \\
\text { b. Estado de resultados } \\
\text { y otro resultado integral. } \\
\text { c. Estado de cambios } \\
\text { en el patrimonio. } \\
\text { d. Estado de flujos de } \\
\text { efectivo. } \\
\text { e. Notas. }\end{array}$ & $\begin{array}{l}\text { a. Estado de situación } \\
\text { financiera. } \\
\text { b. Estado de resultados } \\
\text { integral o un estado de } \\
\text { resultados separado y } \\
\text { un estado de resultados } \\
\text { integral. } \\
\text { c. Estado de cambios } \\
\text { en el patrimonio } \\
\text { d. Estado de flujos de } \\
\text { efectivo. } \\
\text { e. Notas. }\end{array}$ & $\begin{array}{l}\text { a. Estado de situación } \\
\text { financiera. } \\
\text { b. Estado de resultados. } \\
\text { c. Notas. }\end{array}$ \\
\hline Decreto 2420 de 2015 & $\begin{array}{l}\text { Anexo compilatorio } \\
\text { No. } 1 \text { actualizado }\end{array}$ & $\begin{array}{l}\text { Anexo compilatorio } \\
\text { No. } 2\end{array}$ & Anexo No. 3 \\
\hline Referencia técnica & $\begin{array}{l}\text { NIC 1- Presentación } \\
\text { de estados financieros, } \\
\text { párrafo } 10 .\end{array}$ & $\begin{array}{l}\text { Sección 3. Presentación } \\
\text { de estados financieros, } \\
\text { párrafo } 17 .\end{array}$ & $\begin{array}{l}\text { Capítulo } 3 . \\
\text { Presentación de estados } \\
\text { financieros, párrafo } 8 .\end{array}$ \\
\hline
\end{tabular}

Fuente: elaboración propia.

\subsubsection{Administradores de empresas}

La Ley 60 de 1981 (Congreso de Colombia, 1981 ) reconoce la disciplina de Administración de Empresas como profesión y dicta las normas sobre el ejercicio de la misma en Colombia; el artículo 4 estipula que para ejercerla se debe contar con título universitario expedido por una institución de educación superior aprobada por el gobierno nacional y con matrícula profesional que expide el Consejo Profesional de Administración de Empresas ${ }^{5}$.

4 Una empresa que aplique el marco normativo del grupo 2, si en el patrimonio solo presenta cambios por el resultado del período, pago de dividendos, correcciones de errores de períodos anteriores y cambios de políticas contables, se podrá presentar un único estado de resultados y ganancias acumuladas, en vez del estado del resultado integral y del estado de cambios en el patrimonio. Lo anterior es definido en la Sección 6, Estado de Cambios en el Patrimonio y Estado de Resultados y Ganancias Acumuladas.

5 Cabe indicar que para Molina (2006), el concepto de administrador que es abordado en la Ley 60 de 1981 (Congreso de Colombia, 1981) en mención invoca cuestiones funcionales, como que dentro del mismo hacen parte "[...] todas y cada una de las personas
No obsta agregar que la mencionada ley no establece que se requiera tal condición -profesional en Administración de Empresas- para ostentar la calidad de representante legal, excepto que los estatutos, disposición interna o convocatoria para proveer ciertos cargos lo determine. Es de esperarse que para algunos cargos de administración o dirección empresarial se cuente con formación en esta disciplina.

\subsubsection{Contadores públicos y revisores fiscales}

La Ley 145 de 1960 (Congreso de Colombia, 1960) reglamentó el ejercicio de la profesión de contador público y la Ley 43 de 1990 (Congreso de la República, 1990) adiciona y modifica la anterior, definiendo el artículo 1 de esta última, al contador como "la persona

que, al interior o en relación con la sociedad, cumplen funciones de administrador"(p. 63); no obstante, el concepto abordado en el presente artículo es desde el aspecto legal, contemplado en el artículo 22 de la Ley 222 de 1995 (Congreso de Colombia, 1995). 
natural que, mediante la inscripción que acredite su competencia profesional en los términos de la presente Ley, está facultada para dar fe pública de hechos propios del ámbito de su profesión, dictaminar sobre estados financieros $[\ldots]^{\prime \prime}$

Además se estableció que los contadores públicos acreditarán su profesión por medio de la expedición de la tarieta profesional por parte de la Junta Central de Contadores ${ }^{6}$, la cual actuará como tribunal disciplinario.

Actualmente esta profesión cuenta con el Código de ética profesional consagrado en el capítulo cuarto, título primero de la Ley 43 de 1990 (Congreso de la República, 1990). El artículo 35 determina respecto a la disciplina de la Contaduría Pública que es una profesión que debe satisfacer los requerimientos de la sociedad y que son depositarios de la confianza pública.

Igualmente el Código de Comercio expedido en 1971 (Presidencia de la República, 1971), incluyó en el capítulo VIII del Libro II, lo correspondiente al revisor fiscal; allí se encuentra que el artículo 203 estipula las sociedades obligadas a tener dicha figura. También, la Ley 43 de 1990 (Congreso de la República, 1990), en el parágrafo segundo del artículo 13, menciona que será obligatorio tener revisor fiscal en todas las sociedades comerciales, de cualquier naturaleza, cuyos activos brutos al 31 de diciembre del año inmediatamente anterior sean o excedan el equivalente de cinco mil salarios mínimos y/o cuyos ingresos brutos durante el año inmediatamente anterior sean o excedan al equivalente a tres mil salarios mínimos.

6 La Junta Central de Contadores fue creada mediante acto legislativo 2373 de 1956, entidad adscrita al Ministerio de Educación; posteriormente, la Ley 1151 de 2007 la vincula al Ministerio de Comercio, Industria y Turismo y la reconoce como Unidad Administrativa Especial (UAE) del gobierno.
Dentro de las funciones de la revisoría fiscal, contenidas en el artículo 207 del Código de Comercio, se resaltan en materia de contabilidad: la de velar por que se lleve regularmente la contabilidad de la sociedad y autorizar con su firma cualquier balance que se haga, con su dictamen o informe correspondiente.

Como se puede evidenciar, el revisor fiscal emite opinión respecto del cumplimiento de las obligaciones que, en materia de contabilidad y presentación de estados financieros, tienen las organizaciones.

\section{METODOlOGía}

El tipo de estudio es exploratorio (Hernández Sampiere, 2014) y permite abordar un tema poco analizado desde las universidades que tienen programas académicos de Administración de Empresas y Contaduría Pública, como lo es indagar respecto al conocimiento de los estudiantes de dichos programas, acerca de la responsabilidad en la preparación y presentación de los estados financieros en sociedades comerciales.

La población del estudio comprende los estudiantes de los programas académicos de Administración de Empresas y Contaduría Pública de dos universidades de la ciudad de Cali, que desde el año 1975 crearon los programas mencionados. Por confidencialidad se nombrará la Universidad A y Universidad B a cada una de las instituciones en que se aplicó la presente investigación.

La población objeto de estudio estuvo integrada por: a. Los estudiantes de los programas académicos de Administración de Empresas y Contaduría Pública, matriculados en el primer semestre de 2017 de la Universidad A; y b. los estudiantes matriculados en los programas académicos en mención en el segundo semestre del 2017 de la Universidad B. La tabla 2 
presenta la población total de 2.395 estudiantes, que para el caso de los de Administración de Empresas fueron incluidos a partir del tercer semestre de estudios, dado que en ese momento habrían tomado asignaturas del área de desarrollo académico contable y financiero; en tanto que para los de Contaduría Pública, a partir del segundo semestre del plan de estudios.

Tabla 2. Población total caso de estudio.

\begin{tabular}{|c|c|c|c|}
\multicolumn{1}{|c|}{ Programa Académico } & Universidad A & Universidad B & Total \\
\hline Administración de Empresas & 575 & 442 & 1017 \\
\hline Contaduría Pública & 863 & 515 & 1378 \\
\hline Total & 1438 & 957 & 2395 \\
\hline
\end{tabular}

Fuente: elaboración propia.

En la tabla 3 se muestra la distribución general, presentando la Universidad A una participación de 59,96\% y la Universidad B, 40\%.

Siendo la población total de 2.395 estudiantes de los programas de Contaduría Pública y Administración de Empresas de ambas universidades, se calculó el tamaño de la muestra utilizando el muestreo estratificado con asignación proporcional por cada uno de los estratos (Lind et al., 2015), utilizando un margen de error máximo del 3,8\% y un nivel de confianza de $95 \%$, lo que dio como resultado una muestra de 522 estudiantes, distribuidos según la tabla 3 en forma proporcional por universidad y por programa de estudio.

Tabla 3. Distribución general de la muestra por universidad y programa de estudio.

\begin{tabular}{|c|c|c|c|}
\multicolumn{1}{|c|}{ Muestra } & Universidad A & Universidad B & Total \\
\hline Administración de Empresas & 125 & 97 & 222 \\
\hline Contaduría Pública & 188 & 112 & 300 \\
\hline Total & 313 & 209 & 522 \\
\hline
\end{tabular}

Fuente: elaboración propia.

A nivel teórico se consideraron las siguientes ecuaciones:

$$
n=\frac{\sum_{i=1}^{i=h} P_{i}\left(1-P_{i}\right) N_{i}}{N \frac{\varepsilon^{2}}{Z_{\frac{\alpha}{2}}^{2}}+\sum_{i=1}^{i=h} P_{i}\left(1-P_{i}\right) N_{i}}
$$

(2) $n_{i}=n \cdot \frac{N_{i}}{N}$
Donde,

$n_{i}=$ Es el tamaño de la muestra en el estrato

$n=$ Es el tamaño de la muestra

$P_{i}=$ Es la proporción del estrato

$P_{i}\left(1-P_{i}\right)=$ Es la varianza del estrato

$N_{i}=$ Es el tamaño de la población del estrato

$\varepsilon=$ Margen de error en la estimación

$Z_{\frac{\alpha}{2}}^{2} \equiv$ Es el cuantil de la distribución normal estándar asociada al nivel de confianza $(1-\alpha)$

$h=$ Es el número de estratos en que se dividió la población 
La información fue recolectada mediante un cuestionario con preguntas cerradas, que fueron: ¿quiénes tienen la condición legal de ser administradores en una organización?, ¿conoce usted de qué trata la Ley 222 de 1995?, ¿quién es el responsable de los estados financieros?, ¿quiénes deben certificar los estados financieros? y ¿qué tan importante es la certificación de los estados financieros?
Como se pretendía conocer en qué nivel de la formación los estudiantes aprendieron el concepto estudiado, se tomó la población por semestre, se distribuyó de manera proporcional de la muestra en cada semestre por programa de estudio y se aplicó en forma aleatoria.

Una vez identificados los conceptos para la construcción del cuestionario, fue validado con docentes expertos en la temática adscritos a las dos universidades objeto de estudio, comprobando así la pertinencia de cada uno de los interrogantes; posteriormente fue sometido a prueba piloto con estudiantes de los programas académicos de administración de empresas y contaduría pública, a fin de revisar y mejorar el orden metodológico del instrumento; así mismo la fiabilidad en cuanto a la consistencia interna fue realizada mediante el cálculo de los coeficientes alpha de Cronbach (Escofet, Folguerias, Luna, \& Palou, 2016).

La información fue recolectada mediante un cuestionario con preguntas cerradas, que fueron: ¿quiénes tienen la condición legal de ser administradores en una organización?, ¿̇conoce usted de qué trata la Ley 222 de 1995?, ¿quién es el responsable de los estados financieros? ¿quiénes deben certificar los estados financieros? y ¿̇qué tan importante es la certificación de los estados financieros?

\section{RESULTADOS}

\subsection{ADMINISTRADORES EN UNA ORGANIZACIÓN}

La tabla 4 presenta los resultados del interrogante sobre el conocimiento de quiénes tienen la condición legal de ser administradores en una organización. De los encuestados, 77,6\% respondieron correctamente que consideran al representante legal, 29,5\% al suplente del representante legal, $22,6 \%$ al miembro de junta directiva y $13,6 \%$ a los miembros de la junta de socios. 
Tabla 4. Conocimiento de los administradores en una sociedad mercantil por universidad y programa académico en frecuencia y porcentaje.

\begin{tabular}{|c|c|c|c|c|c|c|c|c|c|}
\hline \multirow{2}{*}{ Opciones de Respuesta } & \multicolumn{4}{|c|}{ Universidad A } & \multicolumn{4}{|c|}{ Universidad B } & \multirow{2}{*}{$\begin{array}{c}\text { Total } \\
\text { Frecuencia }\end{array}$} \\
\hline & $A E$ & $\%$ & $C P$ & $\%$ & $A E$ & $\%$ & $C P$ & $\%$ & \\
\hline Representante Legal & 98 & $78 \%$ & 148 & $79 \%$ & 76 & $40 \%$ & 83 & $74 \%$ & 405 \\
\hline $\begin{array}{l}\text { Suplente Representante } \\
\text { Legal }\end{array}$ & 46 & $37 \%$ & 57 & $30 \%$ & 22 & $12 \%$ & 29 & $26 \%$ & 154 \\
\hline Gerente Principal & 99 & $79 \%$ & 117 & $62 \%$ & 72 & $38 \%$ & 69 & $62 \%$ & 357 \\
\hline Suplente Gerente & 48 & $38 \%$ & 55 & $29 \%$ & 29 & $15 \%$ & 29 & $26 \%$ & 161 \\
\hline Miembro Junta Directiva & 27 & $22 \%$ & 27 & $14 \%$ & 27 & $14 \%$ & 37 & $33 \%$ & 118 \\
\hline Miembro Junta de Socios & 18 & $14 \%$ & 19 & $10 \%$ & 13 & $7 \%$ & 21 & $19 \%$ & 71 \\
\hline Contador & 8 & $6 \%$ & 45 & $24 \%$ & 6 & $3 \%$ & 30 & $27 \%$ & 89 \\
\hline Revisor Fiscal & 0 & $0 \%$ & 9 & $5 \%$ & 7 & $4 \%$ & 8 & $7 \%$ & 24 \\
\hline
\end{tabular}

Fuente: elaboración propia.

Conforme se presenta en la figura 1, considerando el total de las opciones de respuestas, los contadores de la Universidad B acertaron en $19,43 \%$ y los de la Universidad A con $30,67 \%$, mientras que los administradores de la Universidad B acertaron en 17,33\% de los casos y los de la Universidad A en $24,37 \%$.

Figura 1. Conocimiento de los administradores en una sociedad mercantil por programa académico y universidad en porcentaje.

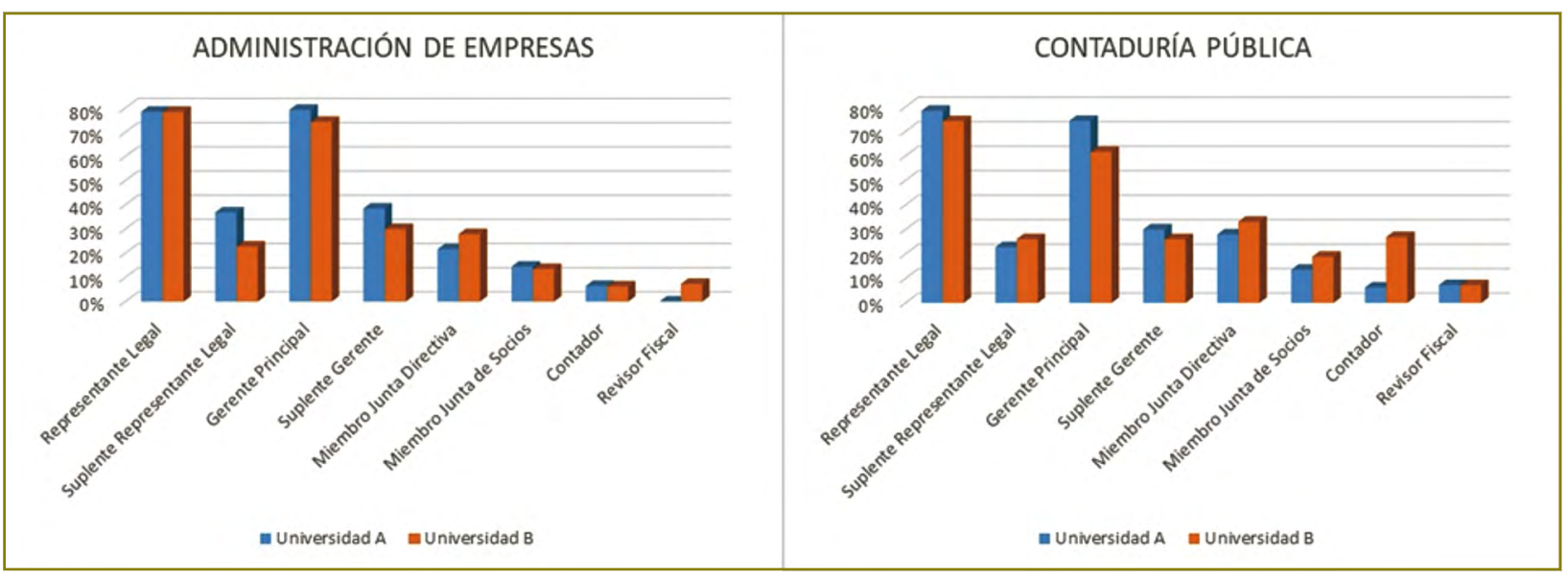

Fuente: elaboración propia.

\subsection{RESPONSABLE DE LOS ESTADOS FINANCIEROS}

La tabla 5 y la figura 2 presentan el resultado del interrogante sobre el conocimiento de quién es el responsable de los estados financieros. Se observa que solo 149 estudiantes consideran al administrador y 359 al contador; desde el aspecto legal, este último no tiene dicha responsabilidad. Así pues, en la Universidad A en el programa académico de Administración de Empresas se identifica que solo 15\% considera que es el administrador y en Contaduría Pública, 35\%; para los encuestados de la Universidad B, el porcentaje es mayor, con $21 \%$ para Administración de Empresas y $40 \%$ en Contaduría Pública. 
Tabla 5. Conocimiento de los responsables de los estados financieros por universidad y programa académico en frecuencia y porcentaje.

\begin{tabular}{|l|c|c|c|c|c|c|c|c|c|}
\hline \multirow{2}{*}{ Opciones de Respuesta } & \multicolumn{4}{|c|}{ Universidad A } & \multicolumn{4}{c|}{ Universidad B } & Total \\
\cline { 2 - 13 } & $A E$ & $\%$ & $C P$ & $\%$ & $A E$ & $\%$ & $C P$ & $\%$ & Frecuencia \\
\hline Revisor Fiscal & 11 & $9 \%$ & 21 & $11 \%$ & 15 & $15 \%$ & 10 & $9 \%$ & 57 \\
\hline Administrador & 19 & $15 \%$ & 65 & $35 \%$ & 20 & $21 \%$ & 45 & $40 \%$ & 149 \\
\hline Contador & 97 & $78 \%$ & 110 & $59 \%$ & 79 & $81 \%$ & 73 & $65 \%$ & 359 \\
\hline No Sabe & 9 & $7 \%$ & 10 & $5 \%$ & 1 & $1 \%$ & 1 & $1 \%$ & 21 \\
\hline
\end{tabular}

Fuente: elaboración propia.

Figura 2. Conocimiento de los responsables de los estados financieros por universidad y programa académico en porcentaje.



Fuente: elaboración propia.
Al confrontar la respuesta correcta por semestre y por programa académico de cada universidad, se observa, en la figura 3, que en octavo semestre de Administración de Empresas de la Universidad A, 25\% de estudiantes tiene mayor conocimiento acerca del responsable de los estados financieros y en la Universidad B, los de tercero, con 56\%. Para Contaduría Pública, los de tercer semestre de la Universidad A, con $71 \%$ y en la Universidad B se destacan 53\% los de estudiantes que están cursando décimo semestre y trabajo de grado. En términos generales, se observa en ambas universidades y programas académicos que a medida que avanzan en los semestres, los estudiantes no mejoran en esta comprensión.

Figura 3. Conocimiento de los responsables de los estados financieros por semestre de cada universidad y programa académico en porcentaje.

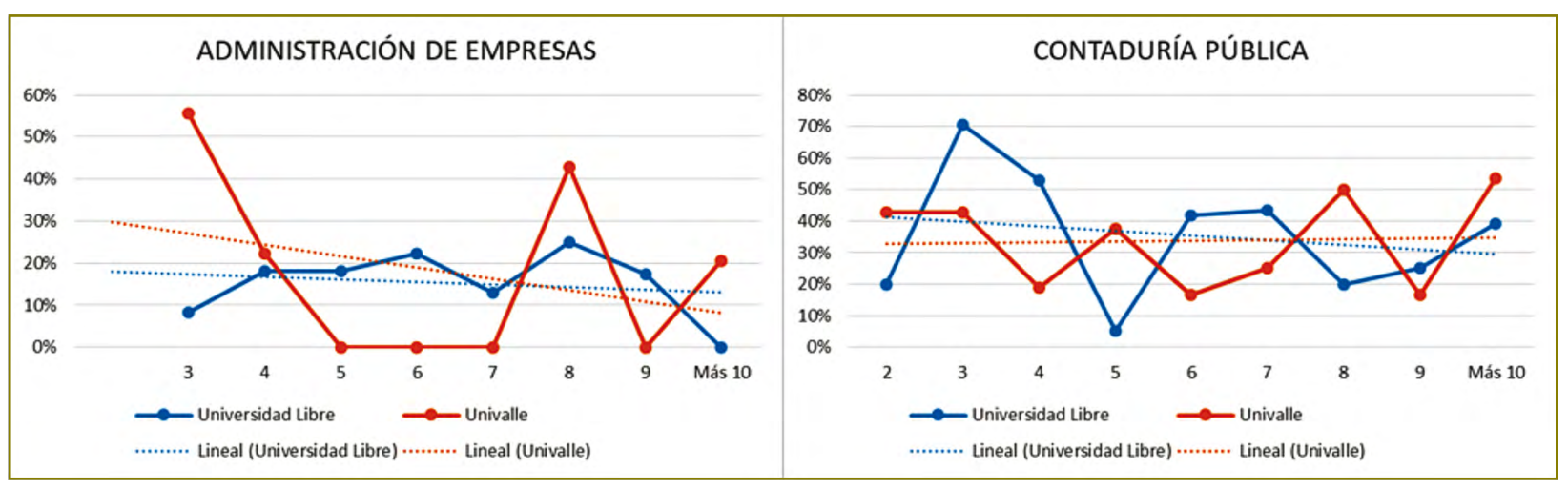

Fuente: elaboración propia. 


\subsection{CERTIFICACIÓN DE ESTADOS FINANCIEROS}

La tabla 6 y la figura 4 presentan el resultado del interrogante sobre quiénes deben certificar los estados financieros. Se observa que solo 23 estudiantes hicieron la combinación correcta, señalando al representante legal (administrador) y contador; al revisar las respuestas en forma individual, 322 indicaron que el administrador y 206 el contador, otros se equivocaron incluyendo al revisor fiscal. Al hacer el análisis por universidad, en la Universidad A, 67\% de los estudiantes de Administración de Empresas señalaron al administrador y $23 \%$ al contador; los de Contaduría Pública marcaron 60\% al administrador y $37 \%$ al contador. Por su parte, en la Universidad B en el programa de Administración de Empresas marcaron $61 \%$ y $42 \%$ al administrador y contador, respectivamente, y en Contaduría Pública 59\% y $60 \%$.

Tabla 6. Conocimiento de los encargados de certificar estados financieros en frecuencia y porcentaje.

\begin{tabular}{|l|c|c|c|c|c|c|c|c|c|}
\hline \multirow{2}{*}{ Opciones de Respuesta } & \multicolumn{4}{|c|}{ Universidad A } & \multicolumn{4}{c|}{ Universidad B } & Total \\
& $A E$ & $\%$ & $C P$ & $\%$ & $A E$ & $\%$ & $C P$ & $\%$ & Frecuencia \\
\hline Revisor Fiscal & 14 & $11 \%$ & 39 & $21 \%$ & 30 & $31 \%$ & 37 & $33 \%$ & 120 \\
\hline Administrador & 84 & $67 \%$ & 113 & $60 \%$ & 59 & $61 \%$ & 66 & $59 \%$ & 322 \\
\hline Contador & 29 & $23 \%$ & 69 & $37 \%$ & 41 & $42 \%$ & 67 & $60 \%$ & 206 \\
\hline No sabe & 6 & $5 \%$ & 7 & $4 \%$ & 3 & $3 \%$ & 2 & $2 \%$ & 18 \\
\hline Administrador y Contador & 3 & $2 \%$ & 6 & $3 \%$ & 6 & $6 \%$ & 8 & $7 \%$ & 23 \\
\hline
\end{tabular}

Fuente: elaboración propia.

Figura 4. Conocimiento de los responsables de los estados financieros por universidad y programa académico.

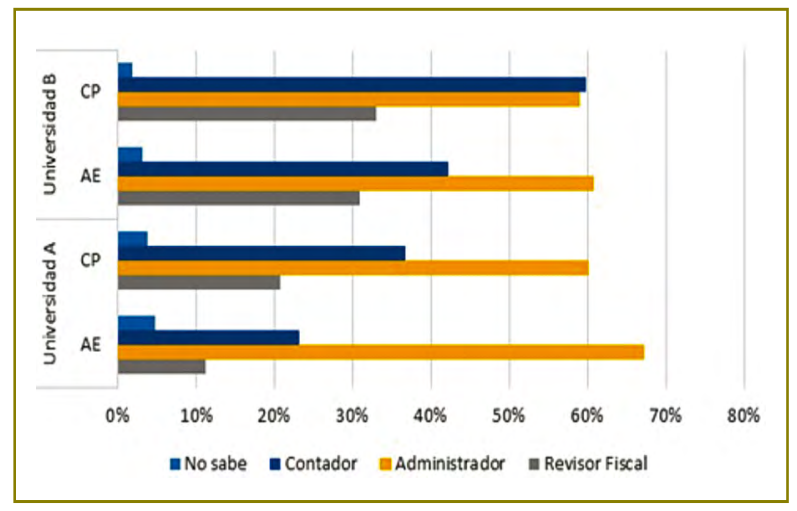

Fuente: elaboración propia.
Al analizar la cantidad de los estudiantes que indicaron que el administrador es uno de los encargados de certificar estados financieros, la figura 5 muestra que en la Universidad A los estudiantes de Administración de Empresas de noveno semestre tienen mayor conocimiento, con $78 \%$, y en la Universidad B los de séptimo, con $83 \%$.

En Contaduría Pública, en la primera universidad mencionada los de quinto y sexto semestres, con $75 \%$, y en la segunda con $75 \%$, los de cuarto y séptimo semestres. Al mirar la tendencia, en la Universidad A se puede considerar que a medida que avanzan, tienden a mejorar y los de la Universidad B se mantienen. 
Figura 5. Estudiantes que marcaron al administrador como una de las personas encargadas de certificar estados financieros por semestre de cada universidad y programa académico en porcentaje.

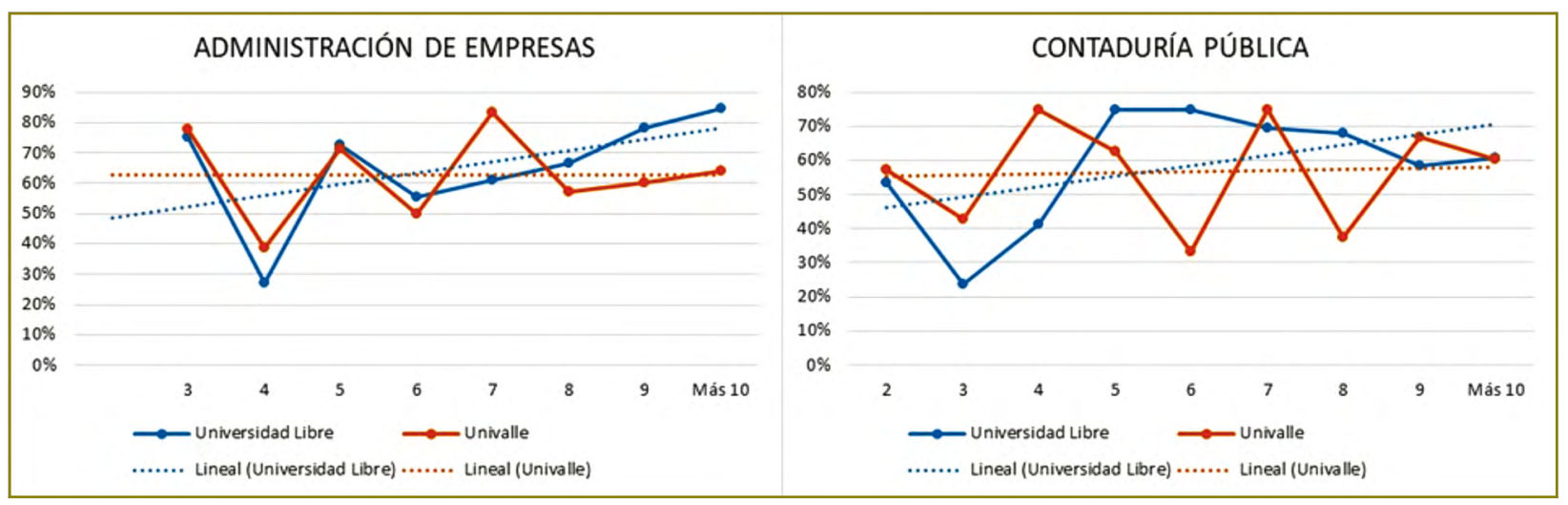

Fuente: elaboración propia.

Al analizar los estudiantes que indicaron que el contador es uno de los encargados de certificar estados financieros, se muestra en la figura 6 que en la Universidad A los estudiantes de Administración de Empresas de cuarto semestre tienen mayor conocimiento, con $45 \%$ y en Contaduría Pública los de tercero, con $53 \%$.

Figura 6. Estudiantes que respondieron que el contador es una de las personas encargadas de certificar estados financieros por semestre de cada universidad y programa académico en porcentaje.

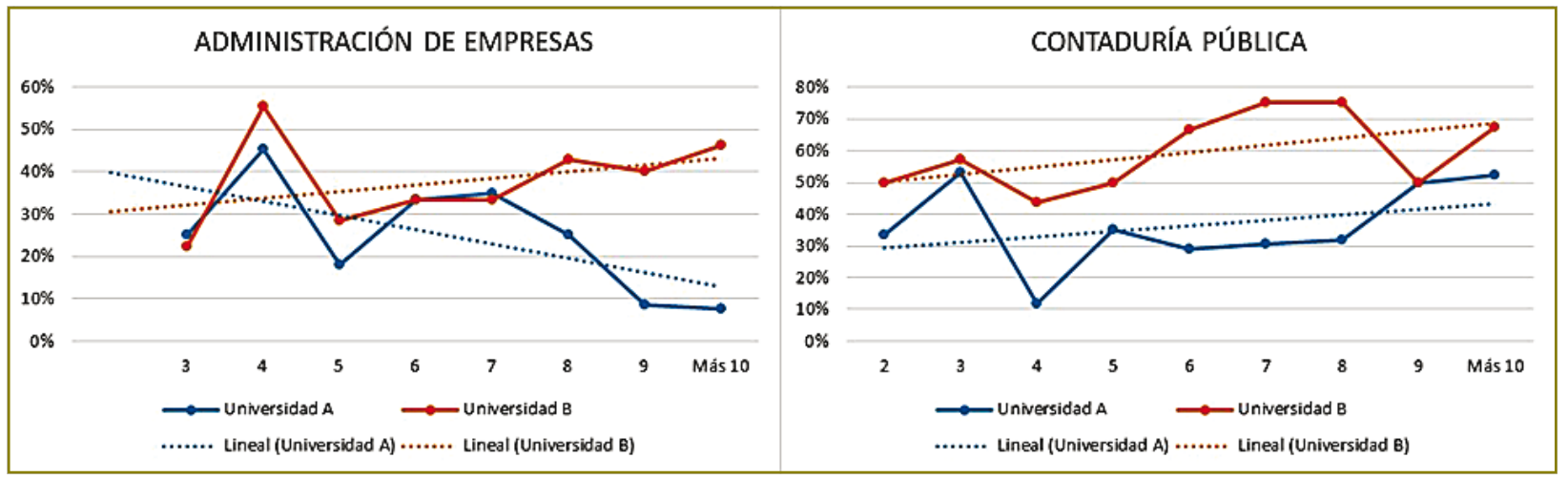

Fuente: elaboración propia.

En la Universidad B, en el programa académico de Administración de Empresas, los estudiantes de cuarto semestre, con 56\%, y en Contaduría Pública, con 75\%, los estudiantes de séptimo y octavo semestres. Al mirar la tendencia, en la universidad A se puede considerar que, a medida que avanzan, los estudiantes de Administración de Empresas no tienen claridad al respecto de preguntado, y en forma contraria los de contaduría pública; mientras que para la Universidad B se observa que la tendencia es a mejorar a medida que avanzan los semestres. 


\subsection{IMPORTANCIA DE LA CERTIFICACIÓN DE ESTADOS FINANCIEROS}

La tabla 7 y la figura 7 presentan las respuestas a la pregunta sobre la importancia de la certificación de los estados financieros. Se encontró que 292 estudiantes consideraron que era obligatoria, y los demás no tienen claridad de la misma debido a que 100 consideran que es opcional, 76 que no es necesaria y 54 no saben. Al revisar la opción correcta por universidad, se evidencia que en la Universidad A, 54\% y 55\% de los estudiantes de Administración de Empresas y Contaduría Pública, respectivamente, indicaron que es obligatoria; y para los de la B, 62\% y $54 \%$ de los encuestados de Administración de Empresas y Contaduría Pública.

Tabla 7. Conocimiento de la importancia de la certificación de estados financieros en frecuencia y porcentaje.

\begin{tabular}{|l|c|c|c|c|c|c|c|c|c|}
\hline \multirow{2}{*}{ Opciones de Respuesta } & \multicolumn{4}{|c|}{ Universidad A } & \multicolumn{4}{c|}{ Universidad B } & Total \\
& $A E$ & $\%$ & $C P$ & $\%$ & $A E$ & $\%$ & $C P$ & $\%$ & Frecuencia \\
\hline Obligatoria & 68 & $54 \%$ & 103 & $55 \%$ & 60 & $62 \%$ & 61 & $54 \%$ & 292 \\
\hline Opcional & 33 & $26 \%$ & 36 & $19 \%$ & 15 & $15 \%$ & 16 & $14 \%$ & 100 \\
\hline No necesaria & 10 & $8 \%$ & 34 & $18 \%$ & 13 & $13 \%$ & 19 & $17 \%$ & 76 \\
\hline No sabe & 14 & $11 \%$ & 15 & $8 \%$ & 9 & $9 \%$ & 16 & $14 \%$ & 54 \\
\hline
\end{tabular}

Fuente: elaboración propia.

Figura 7. Conocimiento sobre la importancia de la certificación de estados financieros por universidad y programa académico en porcentaje.

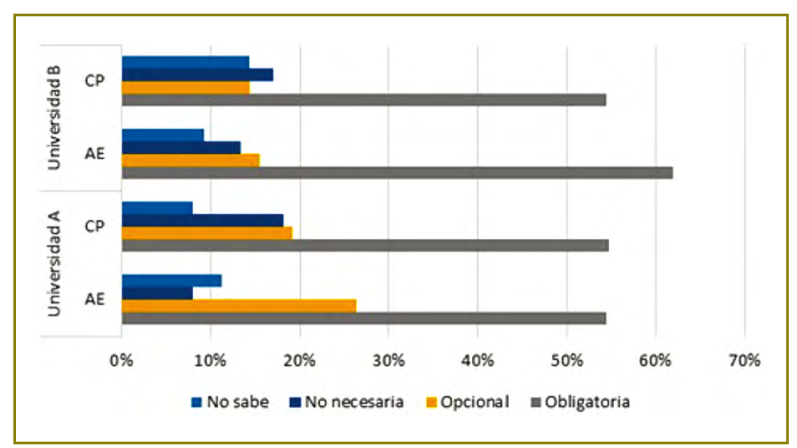

Fuente: elaboración propia.
Al analizar la cantidad de los estudiantes que indicaron que la certificación de los estados financieros es obligatoria, se presenta, en la figura 8, que en la Universidad A los estudiantes de Administración de Empresas de sexto semestre tienen total conocimiento, con $100 \%$, y en la Universidad B, con igual porcentaje, los de séptimo semestre. En Contaduría Pública de la Universidad $A$, los de segundo semestre, con $80 \%$, y en la Universidad B, con $83 \%$, los de sexto y noveno semestres. Al mirar la tendencia, en la Universidad A se puede considerar que desmejoran, y en la Universidad B los de Administración de Empresas se mantienen y los de Contaduría Pública mejoran. 
Figura 8. Estudiantes que marcaron al administrador como una de las personas encargadas de certificar estados financieros por semestre de cada universidad y programa académico en porcentaje.

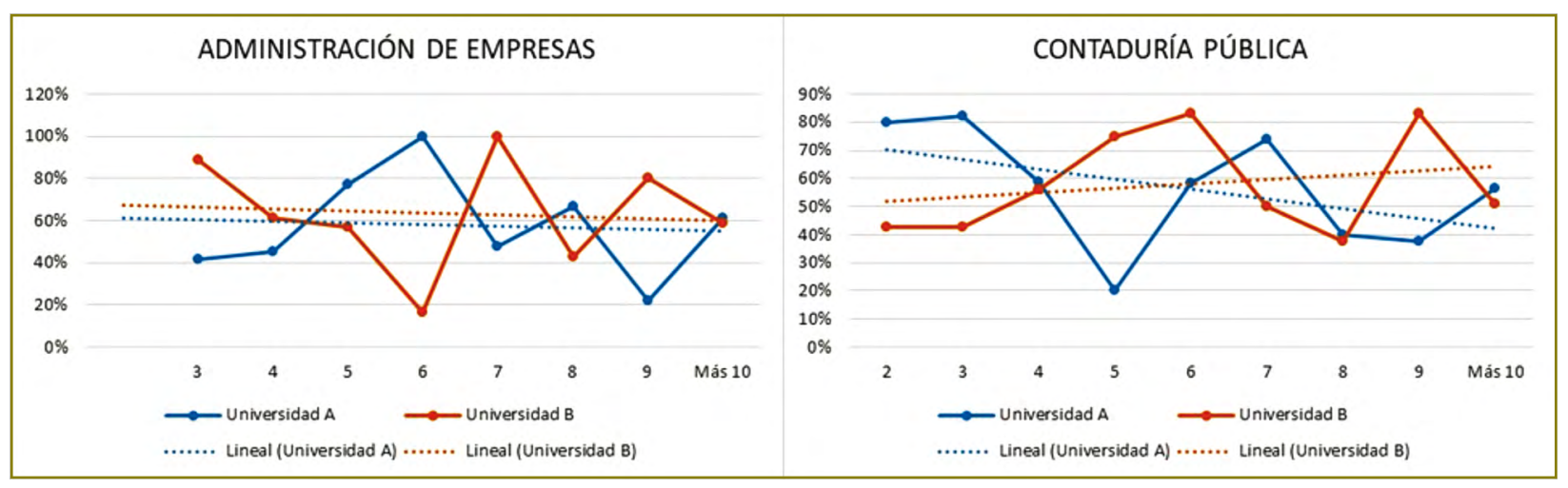

Fuente: elaboración propia.

\section{CONCLUSIONES}

A partir de los resultados que arrojó la investigación se hace evidente la necesidad de que los programas académicos de Administración de Empresas y Contaduría Pública en su contenido curricular y las asignaturas respectivas que ofrecen a sus estudiantes enfaticen la importancia y la responsabilidad que en materia contable y de información financiera les es atribuible $y$, en especial, el efecto que esto tiene frente a los diversos grupos de interés, stakeholders, que basan parte de sus decisiones a partir de los estados financieros con sus respectivas notas. Esta situación da lugar a futuras investigaciones relacionadas con el dominio de los conceptos abordados en este artículo en los profesionales administradores de empresas y contadores públicos, lo cual permitiría identificar oportunidades de formación sobre el alcance y connotación de las responsabilidades que reposan sobre ellos.

Desde el aspecto ético, tanto administradores como contadores deben ser responsables en su actuar profesional; no obstante, las universidades tienen un rol relevante en la formación de estos, difundir el conocimiento y ser muy enfáticos en aquellos temas relevantes en el quehacer de la práctica profesional, como lo son la responsabilidad organizacional, la normatividad legal y la gestión de llevar a cabo satisfactoriamente todas las funciones que les serán asignadas en cada rol que desempeñen en las organizaciones en las que lleguen a ejercer su profesión.

Los administradores deben gestionar adecuadamente el cumplimiento de las políticas y normas contables, de administración de riesgos y de control, mientras que los contadores son los encargados de atender en debida forma el proceso de reconocimiento, medición y revelación en su misión de presentar estados financieros.

Con lo anterior se contribuye no solo a mejorar el desempeño de las organizaciones en que trabajarán y ejercerán en desarrollo de su profesión, sino que, por ser una disposición con rango legal, su inobservancia o equívoca aplicación puede ocasionar posibles sanciones. 


\section{REFERENCIAS}

Aktouf, O. (2001). La administración: entre tradición y renovación. Tercera ed. Cali: Artes Gráficas del Valle.

Alfredo, O., y Becerra, D. (2010). Estado actual de la aplicación de las NIIF en la preparación de estados financieros de las empresas pervanas. Contabilidad y Negocios, 1015), 5-28.

Carolina, Y., y Sánchez, D. (2013). La contabilidad internacional: un enfoque para la presentación de información financiera. Rev. Del Centro de Inv., 39. Pág 33-56.

Cepeda Ulloa, F. (2004). Financiación política y corrupción. Primera ed. Bogotá, D.E.: Planeta.

Congreso de Colombia (1960). Ley 145 por la cual se reglamenta el ejercicio de la profesión de contador público. Bogotá D.C.: Diario Oficial 30433 de febrero 3 de 1961. Recuperado de: https://www.mineducacion.gov.co/1759/ articles-103818_archivo_pdf.pdf

Congreso de Colombia (1981). Ley 60 por la cual se reconoce la Profesión de Administración de Empresas y se dictan normas sobre su ejercicio en el país. Bogotá, D.C.: Diario Oficial 35021 del 23 de noviembre de 1981 . Recuperado de: http://www.mineducacion.gov.co/1621/ articles-103930 archivo pdf.pdf

Congreso de Colombia (1990). Ley 43 Por la cual se adiciona la Ley 145 de 1960, reglamentaria de la profesión de Contador Público y se dictan otras disposiciones. Bogotá: Diario Oficial No. 39602 de 13 de diciembre de 1990. Recuperado de: http://www.mineducacion.gov.co/1621/ articles-104547 archivo pdf.pdf

Congreso de Colombia (1995). Ley 222 Por la cual se modifica el Libro II del Código de Comercio, se expide un nuevo régimen de procesos concursales y se dictan otras disposiciones. Bogotá. Recuperado de: http:// www.secretariasenado.gov.co/senado/ basedoc/ley 0222 1995.html
Consejo Técnico de la Contaduría Pública (2021). Conceptos. Recuperado en marzo 13, 2021, de: https://www.ctcp.gov.co/ conceptos/2021

Congreso de Colombia (2009). Ley 1314. Diario Oficial 47409, del 13 de julio de 2009. Recuperado de: http://historico.presidencia. gov.co/leyes/2009/archivo.html

Presidencia de la República de Colombia (1971). Decreto 410 Por el cual se expide el código de comercio. Bogotá: Diario Oficial No. 33.339 del 16 de junio. Recuperado de: http://www. secretariasenado.gov.co/senado/basedoc/ codigo comercio.html

Epstein, M. J., y Birchard, B. (2001). La empresa honesta. Barcelona: P. Empresa, Ed.)

Escofet, A.; Folguerias, P.; Luna, E., y Palou, B. (2016). Elaboración y validación de un cuestionario para la valoración de proyectos. Universidad del Valle - Colombia. Revista Mexicana de Investigación Educativa RMIE, 21(70), 929-949. Recuperado de: https:// eds-a-ebscohost-com.bd.univalle.edu.co/eds/ pdfviewer / pdfviewer? vid=0\&sid=4020807de b $38-4575$ - b d d 0 094002a $18 \mathrm{e} 03 \% 40$ sessionmgr4008

Garza Sánchez, H. H.; Cortez Alejandro, K. A.; Méndez Sáenz, A. B., y Rodríguez García, M. del P. (2017). Efecto en la calidad de la información ante cambios en la normatividad contable: caso aplicado al sector real mexicano. Contaduria y Administracion, 62(3), 746-760. https://doi.org/10.1016/i. cya.2015.11.012

Hernández Sampiere, R. (2014). Metodología de la Investigación. Sexta ed. México, D.F.: McGraw-Hill Educación. Recuperado de: http://www.ebooks7-24.com/onlinepdfis/ view.aspx

Jensen, M. C.; Meckling, W. H.; Benston, G.; Canes, M.; Henderson, D.; Leffler, K.; Zimmerman, J. (1976). Theory of the Firm: Managerial Behavior, Agency Costs and Ownership Structure. Journal of Financial 
Economics, 3(4), 305-360. Recupedo de: http://ssrn.com/abstract=94043

Lind, D. A.; Marchal, W. G.; Wathen, S. A.; Rubio Ruiz, R. M.; Vizcaíno Díaz, O., y Mason, R. D. (2015). Estadística aplicada a los negocios y la economía. Recuperado de: http://www. ebooks7-24.com.bd.univalle.edu.co/book. aspx?i=2266

Mejía Soto, E.; Montilla Galvis, O. de J.; Montes Salazar, C. A., y Mora Roa, G. (2014). Teoría Tridimensional de la Contabilidad. Primera ed. Pereira: U. Libre.

Molina Arrubla, C. M. (2006). Responsabilidad penal de los administradores de empresas. Segunda ed. Medellín: Universidad Católica de Colombia.

Montaño Orozco, E., y Rendón, Á. B. (2017). Contabilidad: reconocimiento, medición y revelación, más allá del registro. Primera ed. Cali: Universidad del Valle.

Montes Salazar, C. A.; Mejía Soto, E., y Valencia Salazar, J. J. (2006). Paradigmas en Contabilidad. Primera ed. Cali: Artes Gráficos Ltda. Recuperado de: http://www. eutimiomejia.com/portal/images/textos/pdf/ LIBRO PARADIGMA EN CONTABILIDAD.pdf

Murillo Vargas, G.; Zapata, D. Á.; Martínez Crespo, J.; Avila Dávalos, H.; Salas Páramo, J., y López Arellano, H. (2011). Teorías Clásicas de la Organización y el Management. Segunda re. Cali.
OCDE (2004). Principios de Gobierno Corporativo de la OCDE. París: Ministerio de Economía y Hacienda para la edición española. Recuperado de: https://www.oecd.org/daf/ca/ corporategovernanceprinciples/37191543. pdf

Pereda, T. (1990). Algunas implicaciones del paradigma de utilidad en la disciplina contable. Contaduría Universidad de Antioquia, 16.

Rafael, L. F. (2007). Revista del Centro de Investigación. Universidad La Salle, Información Financiera en las PYMES, 7(27), 67-75. https:// doi.org/10.26457/recein.v7i27.231

Ryan, B.; Scapens, R. W., y Theobald, M. (2004). Metodología de la investigación en finanzas y contabilidad. Segunda ed. España.

Sunder, S., y Tr. y rev. tecnica Garcia Nohora et al. (1944). Teoría de la contabilidad y el control. Universidad Nacional de Colombia. 2005. Recuperado de: http://www.fce.unal.edu. co/publicaciones/images/pdf/Teoria de la contabilidad 2015.pdf

Superintendencia de Sociedades, Confecamaras y Cámara de Comercio de Bogotá (2009). Guía colombiana de gobierno corporativo para sociedades cerradas y de familia. Bogotá. Recuperado de: http://www. supersociedades.gov.co/delegatura aec/ informes_publicaciones/responsabilidad social/Cartillas Guias/Guía colombiana de gobierno corporativo.pdf 